\title{
Nicholas Smith, Towards a Phenomenology of Repression - A Husserlian Reply to the Freudian Challenge
}

Acta Universitatis Stockholmiensis, Stockholm Studies in Philosophy 34, Stockholm University, 2010, 342 p.

\section{Natalie Depraz}

\section{(2) OpenEdition}

\section{Journals}

Édition électronique

URL : http://journals.openedition.org/alter/1440

DOI : $10.4000 /$ alter. 1440

ISSN : 2558-7927

Éditeur :

Association ALTER, Archives Husserl (CNRS-UMR 8547)

Édition imprimée

Date de publication : 1 octobre 2011

Pagination : 249-257

ISBN : 978-2-9522374-7-5

ISSN : 1249-8947

\section{Référence électronique}

Natalie Depraz, « Nicholas Smith, Towards a Phenomenology of Repression - A Husserlian Reply to the Freudian Challenge », Alter [En ligne], 19 | 2011, mis en ligne le 01 janvier 2020, consulté le 25 septembre 2020. URL : http://journals.openedition.org/alter/1440 ; DOI : https://doi.org/10.4000/alter. 1440 


\section{NICHOLAS SMITH, TOWARDS A PHENOMENOLOGY OF REPRESSION - A HUSSERLIAN REPLY TO THE FREUDIAN CHALLENGE (ACTA UNIVERSITATIS STOCKHOLMIENSIS, STOCKHOLM STUDIES IN PHILOSOPHY 34, STOCKHOLM UNIVERSITY, 2010, 342 P.)}

\section{Introduction}

Choisir de faire se rencontrer Husserl et Freud autour de la thématique du refoulement (Verdrängung) plutôt que de l'inconscient ou de la pulsion ${ }^{1}$ dénote une connaissance ciblée des deux auteurs. Certes, tous deux utilisent ce concept, même s'il est plus central chez Freud, mais il y a quelque originalité à susciter une telle confrontation: " the present work investigates the possibility for transcendantal phenomenology to clarify Freud's concept of the unconscious, with a focus on theory of repression. » (p. 1) L'orientation choisie est donc clairement "phénoménologique ", comme le titre l'indique: «Towards a phenomenology of repression ». Plus spécifiquement, la méthode adoptée consiste à donner la primeur à la phénoménologie comme éclairage critique de l'approche freudienne, et non à produire une enquête comparative entre les deux. Assurément, la lecture de la phénoménologie husselienne proposée se trouve infléchie du fait de l'objet de l'enquête. Pour éclairer le fonctionnement inconscient $\mathrm{du}$ refoulement freudien par le projecteur de la phénoménologie, N. Smith met l'accent sur la phénoménologie génétique et, plus particulièrement, sur l'expérience de la passivité et du présent vivant.

Ainsi, du sein d'un cadre général qui met en avant l'aptitude la phénoménologie husserlienne à explorer davantage que le simple "pré-conscient», c'est-à-dire à rendre compte de l'authenticité de

1. Cf. G. Karlsson, Psychoanalysis in a new light, Cambridge University Press, 2010. 
processus inconscients en jeu dans les tendances passives primaires du sujet, l'auteur focalise son attention sur le mécanisme spécifique du refoulement. Le choix d'un phénomène aussi précis peut intriguer, d'autant plus que Freud and Husserl, tout en employant le même terme Verdrängung, l'utilise dans des contextes distincts et à des niveaux différents. L'entente freudienne du refoulement est caractérisée par une forte tension émotionnelle : "Repression, according to Freud, occurs for instance when a lived experience is deemed too emotionally painful to deal with in the same way that normal experiences are dealt with (...) » (p.1) En revanche, la portée husserlienne du phénomène se limite à la dynamique structurelle de l'activité perceptive, faite de différentes dimensions négatives, "conflictuelle ", lorsqu'il y a rencontre d'un objet lato sensu (une chose physique, un évenement, une situation, une personne). Certaines de ces dimensions demeurent cachées, résistent à la vue. Il se peut que je sois incapable de les identifier, de les reconnaître: Husserl parle d'empêchement ou d'obstacle (Hemmung) pour ce qui est de l'objet, de déception ou de frustration (Enttäuschung) du côté du sujet. A première vue, les deux analyses sont situées sur deux plans hétérogènes, comme cela est très clairement montré au chapitre 1 de la première Partie, intitulé "Repression and perception: differing foundations » (pp. 41-73): d'un côté, on est sur le plan de la dynamique inconsciente par émergence et contre-émergence ("resistance », « défense ») de contenus psychiques qui opèrent comme des forces, d'un autre côté sur celui d'une corrélation intentionnelle formelle et structurelle qui relie un objet à l'activité directionnelle de visée d'un sujet. Bref, le premier est pratique et fortement émotionnel, le second essentiellement théorique et cognitif. Pourtant, un examen plus précis montre que le mécanisme de refoulement en jeu de chaque côté fonctionne en structure de façon très affine : dans le deux cas, on a affaire à « an unconscious activity of pushing away something from consciousness while ensuring that it remains efficacious (...) as something foreign within (me). » (p. 1). En fait une telle définition «préliminaire " du refoulement est complexe, elle permet d'identifie une structure commune à Husserl et à Freud formulée à l'aide d'un terme unique: Ichspaltung (auto-division). Ce terme désigne le processus similaire en jeu chez les deux auteurs, même s'il est plus méthodologique chez Husserl en tant que processus de division au sein du soi sujet à une réduction, et plus pathologique chez Freud en tant que scission de l'ego au sein du processus de défense. Une telle définition unifiée de la dynamique du refoulement est identifiée très tôt dans l'ouvrage via la thématique originale de "l'altérité au sein du soi » sur laquelle je vais revenir plus loin. 


\section{Situation}

Quelle est la spécifique de l'approche de N. Smith dans le contexte interprétatif ? Il suffit de se reporter à la troisième section de l'Introduction, "The field of interpretation : overview of previous littérature » (pp. 10-37), pour prendre connaissance du réseau herméneutique dans lequel s'inscrit l'auteur, et qu'il restitue avec clarté, honnêteté et une grande authenticité. Tout en prenant appui sur cette belle synthèse, je ne suivrai pas ici l'ordre chronologique adopté dans l'ouvrage, mais procèderai à une ré-organisation thématique par cercles concentrique, allant du plus critique au plus coopératif.

Premièrement, Smith construit son interpretation contre la lecture phénoménologique standard dans la France d'après-guerre (MerleauPonty, Levinas, Derrida, Ricœur, Henry), selon laquelle Husserl, limitant l'inconscient au pré-conscient, ne serait pas à même de faire droit à la radicalité freudienne de l'altérité (entendue comme sysnonyme de l'inconscient); deuxièmement, l'auteur rend compte du paysage essentiellement germanophone de l'interprétation génétique husserlienne sur trois décennies (années 60 à 90). Depuis une synthèse incisive, il situe sa propre avancée parmi des lecteurs éminents (Held, Holenstein, Landgrebe, Yamaguchi, Lee) et nous offre ce faisant un aperçu brillant des différentes lignes de la recherche sur ce point, $q^{\prime} u^{\prime} i l^{\prime} s^{\prime}$ agisse des lignes de force ou des insuffisances ; troisièmement, une attention spéciale est accordée à l'hypothèse originale de Rudolf Bernet: alors que le premier cercle procure à Smith un puissant contre-levier et que le second donne du relief au champ genétique, l'approche de Bernet fournit le cadre plus large de l'investigation plus locale de l'auteur, cadre clairement résumé ainsi : «engage phenomenology and psychoanalysis in the double and reciprocal movement of a critical self-examination of their own fundamental presuppositions, in order to thereby open a path for a renewed phenomenological analysis of subjective life. » (p. 29) Mas Bernet choisit de conférer à l'imagination un rôle fondateur et exclusif, ce que Smith met en question au nom de son exclusivité unilatérale, proposant de faire davantage droit à la dimension intersubjective ; quatriemement, Smith instaure un dialogue fécond avec les travaux les plus pertinents consacrés à la dernière moûture de l'intersubjectivité husserlienne disponible dans les Hua XIII-IV et XV (1973) (Depraz, Zahavi, Bégout, Römpp, Birnbaum, Montavont, Housset). Son examen est tout à la fois critique et pénétrant, ce qui permet de mesurer plus en profondeur son propre apport: par exemple, son désaccord avec Zahavi concernant la lecture de Derrida et de Freud, l'évaluation positive de Bégout à propos de la description des relations entre le plan statique et le plan génétique. Last but not least, Smith mentionne un article récent (2006) de J. Brudzinska, «Die phänomenologische 
Erfahrung und die Frage nach dem Unbewußten. Überlegungen im Anschluß an Husserl und Freud », qui prend étroitement appui sur Bernet mais va plus loin en "pointing to an 'originary bivalence' between phantasy and perception. The unconscious consequently amounts to a "presencing » situated beneath the distinction between both, which is also named " consciousness of an originary otherness ». (p. 37)

\section{Hypothèse générale et structure de l'ouvrage}

L'ouvrage est sobrement divisé en deux temps. Smith se concentre tout d'abord sur la phénoménologie génétique et ses traits méthodologiques, considérée comme la ressource la plus féconde pour aborder l'inconscient freudien. L'idée est la suivante : la réduction génétique, qui porte davantage sur les «processus de flux » que sur la corrélation entre l'objet and l'acte répond au niveau expérientiel du processus inconscient. Cette hypothèse est tout à fait adaptée, et se trouvé étayée dans l'ouvrage par de nombreuses analyses détaillées des différentes formes de reductions génétiques, intersubjective, apodictique, monde de la vie. La deuxième partie is consacrée à des « rencontres concrètes » avec l'altérité freudienne en jeu dans le procès du refoulement. Quelques thèmes cruciaux sont retenus, qui donnent lieu à une confrontation aiguë : 1. la temporalité de l'inconscient éclairée par la Nachträglichkeit ; 2 . la règle de l'association libre repensée par la synthèse passive ; 3 . La dynamique des pulsions réinvestie depuis l'intentionalité pulsionnelle. La thèse de Smith est la suivante : contrairement à la conception générale de la phénoménologie française d'après-guerre (Merleau-Ponty, Derrida, Ricœur, Sartre aussi, quoique non-mentionné, sauf dans une note p. 10), qui table sur le départ nécessaire d'avec Freud, un nouvel examen des textes de Husserl fait ressortir une affinité troublante avec les processus inconscients (pp. 6-7). Husserl ne peut-il pas résonner avec l'altérité freudienne radicale, à savoir avec l'étrangeté en jeu dans le mécanisme de refoulement, qui témoigne de ma résistance psychique primaire), ne nous offre-t-il qu'une pré-conscience relative toujours conscientisable? Smith contre-argumente: on trouve chez Husserl des ressources génétiques radicales, expérientielles et méthodologiques, qui captent parfaitement la texture et la dynamique de l'inconscient freudien. A cet égard, l'auteur décrit avec force détails les multiples formes d'altérité qui habitent ma réalité psychique : alors que l'altérité du monde et de la personne relèvent d'une altérité intersubjective, $l^{\prime}$ « otherness within me » est une altérité égologique radicale qui prend diverses formes : corporelle, temporelle et imaginative. 


\section{Croisements entre Husserl et Freud}

Au long des trois chapitres de la première Section, Smith construit pas à pas un cadre rigoureux destiné à montrer comment les différentes réductions husserliennes sont à même de rencontrer l'altérité freudienne la plus radicale qui se loge dans le processus inconscient $\mathrm{du}$ refoulement. Le premier niveau concerne l'analyse encore en partie statique des conflits perceptifs liés au vécu de la négation; le second traite de la théorie de la réduction, qui révèle utimement les modes d'altération en jeu dans la scission réfléchissante de la réduction psychologique; le troisième traite du processus radical de temporalisation à l'œuvre dans le flux an-égoïque. A chaque fois, Smith indique comment ces différentes strates d'analyse réductive répondent a une facette de la théorie freudienne du refoulement, à travers, 1) le vécu de la résistance, 2) la dynamique d'étrangeté interne, 3) les processus inconscients primaires d'altération. Creusant à partir de Husserl, l'auteur touche finement les aspects description de l'altérité et de l'altérité internes qui rencontrent à plein les composantes du refoulement: l'analyse phénoménologique n'est pas séparée ni menée en autarcie, mais prépare avec élégance et de façon intrigante la discussion comparative, qui fait l'objet de la deuxième Section. En fait, c'est la vertu de ce qui est nommé une façon «indirecte » de procéder que d'instiller à mesure dans l'esprit du lecteur des gouttelettes freudiennes, de façon à faire émerger une connection co-générative entre l'altérité égoïque interne et l'inconscient psychique. Je trouve que cette manière de faire est très élégante et convaincante: l'auteur forge par ce biais une méthodologie comparative dynamique inédite qui engage une validation mutuelle de l'analyse phénoménologique théorique et de l'analyse empirique psychanalytique. ${ }^{2}$ On y voit opérer de façon remarquable le tissage continu des registres empiriques et transcendantaux au lieu le plus profond du procès d'alteration en jeu dans la dynamique de l'inconscient : à ce point, la suture est accomplie et l'opération disjonctive entre phénoménologie et psychanalyse n'efface d'elle-même, ce qui ouvre la voie au niveau unifié d'approche de la deuxième Section.

Durant la deuxième Section, le lecteur est invité à partager avec l'auteur trois «concrete encounters » entre Freud et Husserl, au long de trois thèmes cruciaux : temporalité, association et pulsions. Ces trois rencontres sont «attendues » : la plus originale est selon moi la seconde, l'association. En effet, la troisieme, quoique pertinente, mais

\footnotetext{
2. Je me permets de noter en passant qu'une telle démarche permet de valider empiriquement l'intuition "altérologique " transcendantale interne en jeu dans N. Depraz, Transcendance et incarnation. L'altérité à soi chez Edmund Husserl, Paris, Vrin, 1995, sur lequel Smith prend explicitement appui.
} 
déjà frayée par Lee et Bernet, est un peu convenue ; la première, également abordée par Bernet, offre une belle image de l'isomorphie plausible du temps de l'inconscient : la temporalité husserlienne, passive, contient des trous et des brèches, ce qui répond tout à fait au temps hétérogène de l'inconscient freudien. De plus, l'opérativité du refoulé trouve une reformulation phénoménologique de Nachträglichkeit. Le chapitre 2, qui traite de l'association, est le plus intéressant: sur la base de l'inscription des affects au sein du processus rétentionnel-protentionnel (ce qui dé-formaliese le temps), l'association opère à la charnière de l'implication intentionnelle, niveau médian où agit églament la loi de l'association libre à la croisée du conscient et de l'inconscient. Mais les phénomène les plus frappants que relève Smith dans ce cadre à l'aide de son microscope freudien, $c^{\prime}$ est d'une part la dynamique d'une force affective qui ne diminue pas dans le temps et qui se nomme Perseveranz, d'autre part l'interpénétration des souvenirs qui construit l'illusion prégnante (Scheinbild) d'un passé qui n'a jamais eu lieu (pp. 201-202 ; p. 228 sq., chapitre 5, §5/6). Des phénomènes atypiques de ce genre sont étonnants et fructueux : ils défont l'image-standard view du présent vivant en faisant s'effondrer l'idée de la durée comme décroissance affective et la notion du passé comme sédimentation. De tels contrephénomènes pourraient apparaître comme des cas isolés ou marginaux (peu intégrables dans une analyse phénoménologique) s'ils ne s'ancraient pas directement dans le présent vivant en le perturbant. A cet égard, ils représentent des avancées originales en vue du renouvellement de la phénoménologie husserlienne et/ou révèlent l'existence possible d'une phénoménologie freudienne dotée de marqueurs husserliens.

\section{Discussion}

Je ne suis pas spécialiste de Freud et je dirai même que ma connaissance de la psychanalyse se limite à la connaissance de quelques textes de base. De plus, mon intérêt pour la psychanalyse est luimême in-formé par le rapport que les phénoménologues (Husserl, Merleau-Ponty, Ricœur, M. Henry) ont entretenu avec elle. A cet égard, Rudolf Bernet, auquel l'auteur se réfère souvent, ${ }^{3}$ occupe une position tout à centrale dans ce croisement entre phénoménologie

\footnotetext{
${ }^{3}$. R. Bernet, «L'analyse husserlienne de l'imagination comme fondement du concept freudien d'inconscient » (1994) ; "The Unconscious between Representation and Drive : Freud, Husserl and Shopenhauer» (1996); "Pulsion, plaisir et déplaisir. Essai d'une fondation phénoménologique des concepts psychanalytiques » (2001); « Unconscious consciousness in Husserl and Freud » (2002).
} 
husserlienne et psychanalyse freudienne. Mes questions resteront pour ce qui me concerne orientées par la phénoménologie.

A propos de certaines référence manquantes: en parcourant les ouvrages de Husserl mentionnés en bibliographie, je remarque que certains sont absents : par exemple, le Hua XXXVIII (2004), qui traite de l'attention et de la perception et qui contient des descriptions précises de la volonté, de l'émotion, du plaisir, de l'intérêt, des pulsions, de l'intensité et de l'énergie, en lien avec les manuscrits sur la structure de la conscience, encore en cours d'édition, où la thématique traitée par Smith est explicitement abordée. Par ailleurs, au niveau des travaux sur Husserl, la référence à Steinbock eût été plus que requise. De façon plus générale, le diagnostic critique relatif à Ricœur est très pertinent: ce dernier considère que la phénoménologie est limitée (l'inconscient se réduit au pré-consciens) et que la profondeur corrélative du mécanisme inconscient du refoulement est inaccessible pour la première (pp. 14-15). A contrario, Smith révèle les potentialités inhérentes à la passivité et à l'intersubjectivité chez Husserl, potentialités que le Ricœur du début ne prend pas en compte. Or, l'ouvrage plus tardif de Ricœur, Soi-même comme un autre (1990) et, plus précisément, le dixième Essai consacré à l'ontologie de l'altérité, n'est pas pris en considération par l'auteur. C'eût eté un recours précieux pour différencier la position de Ricœur : en effet, il y identifie chez Husserl une "alterite intime» directement liée à la passivité (p. 368) ; en outre, de façon intéressante, Ricœur fait aussi appel à Freud un peu plus tard dans le même essai pour décrire l'altérité inhérente à la conscience dans le cas du Surmoi (p. 408).

A propos de la méthodologie: la démarche comparative quoiqu'asymétrique suivie est très claire : la phénoménologie génétique de Husserl est un outil expérientiel qui éclaire l'inconscient freudien et, plus particulièrement, le mécanisme du refoulement. Au sein de cette strategie comparative, l'auteur procède alternativement à des clarifications "indirectes" et "directes", présentées comme des " small speedboats charging ahead of the 'indirect', methodical presentation of genetic phenomenology» (p. 9). Je comprends la signification générale de ces termes "direct», "indirect», et la raison pour laquelle le mode principal de présentation est indirect, étant donné l'usage des concepts husserliens de passivité et de présent vivant en termes de mise en lumière du mécanisme freudien du refoulement. J'ai plus de doutes à l'égard des « approches directes » où sont mobilisés des concepts psychanalytiques que Husserl luimême décrits : folie, anormalité, souvenirs oubliés, maladie psychiques, complexe pulsionnel. La comparaison n'est-elle pas ici un peu extrinsèque ? Ces analyses ne sont-elles pas un tribut que l'auteur se sent obligé de payer à la nécessité exégétique de la relation historique entre deux auteurs? A cet égard, le cœur de l'approche directe, 
pp. 164-172, où Smith se réfère aux manuscrits des années 30 se rapportant explicitement à l'analyse freudienne des pulsions est certes fascinant: on y voit combien ce dernier connaissait de près la psychanalyse, notamment concernant la pratique des pulsions et le phénomène de la cure. Ma question est: en quoi la référence à ces données empiriques est-elle phénoménologique ? Pour prendre une analogie: on trouve de nombreux passages où Husserl aborde les thèmes $\mathrm{du}$ sommeil, du rêve, du sommeil sans rêve et de l'éveil. Cependant, on peut se demander dans quelle mesure ces phénomènes sont alors approchés phénoménologiquement, ou s'ils ne sont pas des fils conducteurs destinés à parler métaphoriquement de l'épochè.

A propos de la pratique de la phénoménologie : l'auteur souligne à plusieurs reprises la nécessité $d$ 'avoir un regard clinique sur Freud : "the unconscious for Freud is not a 'part' of consciousness but something that can only be introduced from within the clinical situation " (pp. 3-4), ce qui va de pair avec la conception de l'inconscient comme hypothèse et non comme axiome. Cet accent clinique manifeste un intérêt fort pour la dimension pratique de la psychanalyse plutôt que pour sa seule théorie, laquelle possède des limites évidentes : rationalité étroite, biologisme rémanent. Je me demande si l'auteur serait prêt à aborder également la phénoménologie sous un angle pratique : l'épochè en tant que suspension des préjugés est-elle affranchie de biais théoriques? Plus radicalement, que signifierait d'adopter un regard "clinique » en phénoménologie, de façon à ne pas être pris au piège des affirmations théoriques ( $\mathrm{y}$ compris la théorie de la réduction, voire celle de la passivité, du présent vivant), et étudier la praxis du travail phénoménologique en tant qu'opération et non comme doctrine ? ${ }^{4}$

Une dernière question concerne le terme freudien de "dénégation »(Verneinung). Même s'il intervient plus tard, en 1925, alors que l'investigation se centre sur les années 1915, on le présente souvent en parallèle de Verdrängung, ce dernier opérant dans le champ conscient/inconscient, le premier à l'articulation de la conscience et du langage. Or, dans Expérience et jugement, Husserl décrit précisément les différents modes de négation (refus, incertitude, hésitation, probabilité, doute) qui sont en jeu dans notre expérience passive et il les présente comme des modalités expérientielles préprédicatives, même si elles sont articulées sur un mode pré-logique aux prédications judicatives. Il nous offre ainsi une ariculation expérientielle entre langage et perception au niveau même de la réceptivité passive. De façon intéressante, cela semble bien être

\footnotetext{
${ }^{4}$ A ce propos, N. Depraz, Comprendre la phénoménologie. Une pratique concrète, Paris, A. Colin, 2006 ; Lire Husserl en phénoménologue. Idées directrices I, Paris, PUF/CNED, 2008 ; Plus sur Husserl : une phénoménologie expérientielle, Paris, Atlande, 2009.
} 
l'orientation de Freud : n'y a-t-il pas un parallèle entre refoulement et dénégation en tant que complexe perceptif/langagier? Etant donné l'intérêt antérieur de l'auteur pour les Recherches logiques, on eût attendu un traitement plus précis de ce niveau d'approche.

Natalie Depraz 